\title{
Электрон-электронное и электрон-фононное взаимодействия в графене на полупроводниковой подложке: простые оценки
}

\author{
(C) С.Ю. Давыдов
}

Физико-технический институт им. А.Ф. Иофрфе Российской академии наук, 194021 Санкт-Петербург, Россия

Санкт-Петербургский национальный исследовательский университет информационных технологий, механики и оптики, 197101 Санкт-Петербург, Россия

E-mail: Sergei_Davydov@mail.ru

(Получена 25 мая 2017 г. Принята к печати 22 июня 2017 г.)

\begin{abstract}
В рамках расширенных моделей Хаббарда и Холстейна-Хаббарда для электрон-электронного и электронфононного взаимодействий рассмотрена задача об эпитаксиальном графене, сформированном на полупроводниковом субстрате. Для плотности состояний подложки выбрана модель Халдейна-Андерсона. Рассмотрены три области фазовой диаграммы: волны спиновой и зарядовой плотностей и однородное по спину и заряду парамагнитное состояние. На примере ряда частных случаев продемонстрированы как сходства, так и различия электронного состояния графена на полупроводниковой и металлической подложках. Показано, что основное различие возникает в случае, когда точка Дирака графена лежит в пределах запрещенной зоны полупроводника. Численные оценки сделаны для $\mathrm{SiC}-$-подложки.
\end{abstract}

DOI: 10.21883/FTP.2018.03.45621.8658

\section{1. Введение}

Влиянию кулоновского отталкивания на электронный спектр свободного графена (СГ) посвящено значительное число работ (см., например, [1-8]). При этом популярной является стандартная модель Хаббарда $(\mathrm{HM})$, в которой учитывается внутриатомная корреляция электронов $U$ (одноцентровое отталкивание). Используется и расширенный вариант хаббардовской модели (ЕНМ), где дополнительно включено межатомное взаимодействие электронов $G$ (межцентровое отталкивание). В рамках этих моделей удается определить области параметров, отвечающие однородному состоянию дираковского полуметалла (ПМ), в котором каждому атому углерода соответствует один электрон с нулевым результирующим спиновым моментом, и состояниям с неоднородным спиновым и зарядовым распределением электронов по подрешеткам, или о волнах спиновой плотности (ВСП) и зарядовой плотности (ВЗП). В отличие от бесщелевого электронного спектра дираковского ПМ, ВСП и ВЗП состояния в свободном графене обладают щелью и являются моттовскими изоляторами.

В работе [9] ЕНМ была использована для описания эпитаксиального графена (ЭГ) на металлической подложке и определения границ ПМ, ВСП и ВЗП областей. Было, в частности, показано, что, в отличие от СГ, для ЭГ на металле в ВСП и ВЗП состояниях щели (как области запрещенных состояний) отсутствуют, а в ПМ состоянии электронный спектр в окрестности точки Дирака нелинеен и плотность состояний никогда не обращается в нуль. Причина в том, что плотность состояний, отвечающая зоне проводимости металла, конечна во всем энергетическом диапазоне.
В настоящей работе EHM [9] применена к описанию однослойнойного графена на полупроводнике. При этом мы будем использовать также результаты работы $[10]$, где изучалась электронная структура графеноподобных соединений на полупроводниковом субстрате. Дело в том, что учет электронных корреляций в графене приводит в общем случае к различию чисел заполнения $n_{A}$ и $n_{B}$ подрешеток $A$ и $B$ графена. Та же ситуация $\left(n_{A} \neq n_{B}\right)$ имеет место и в графеноподобных бинарных соединениях $A_{N} B_{8-N}$ в силу того, что подрешетки $A$ и $B$ заняты химически различными атомами. Отметим, что неравенство $n_{A} \neq n_{B}$ отвечает присутствию щели в электронном спектре и плотности состояний. Таким образом, при наличии ВСП или ВЗП графен формально эквивалентен графеноподобному соединению.

Для исследования влияния электрон-фононного взаимодействия удобна модель Холстейна-Хаббарда (НHМ), где учитывается и электрон-электронное (как правило, только одноцентровое) отталкивание [11-20]. ННМ применяется к описанию сверхпроводимости (в том числе, высокотемпературной), ВСП и ВЗП, поляронов и биполяронов в $3 D-, 2 D$ - и $1 D$-решетках, а также кластерах.

В работе [21] предложена модель для ЭГ на металле, объединяющая ЕНМ и модель Холстейна (расширенная модель Холстейна-Хаббарда - ЕННМ), описывающую взаимодействие зонного электрона с эйнштейновским фононом. Как и в [9], рассмотрены три области фазовой диаграммы: волны спиновой и зарядовой плотностей (ВСП и ВЗП) и однородное по спину и заряду состояние. Здесь мы экстраполируем результаты работы [21] на случай полупроводниковой подложки. 
Таким образом, цель настоящей работы состоит в выяснении следующего вопроса - как появление запрещенной зоны в электронном спектре подложки влияет на состояния ЭГ при условии, что эти состояния формируются не только за счет кинетического обмена ( $t$-взаимодействие, см. далее), но также электронных корреляций и электрон-фононной связи.

\section{2. Электрон-электронное отталкивание}

\section{1. Общие соотношения}

Как показано в [9], в приближении Хартри-Фока диагональные функции Грина ЭГ для подрешеток $A$ и $B$ в модели ЕНМ имеют следующий вид:

$$
G_{A A(B B)}^{\sigma}(\omega, \mathbf{k})=\frac{\Omega_{B(A)}^{\sigma}+i \Gamma(\omega)}{\left(\Omega_{A}^{\sigma}+i \Gamma(\omega)\right)\left(\Omega_{B}^{\sigma}+i \Gamma(\omega)\right)-t^{2} f^{2}(\mathbf{k})},
$$

$f(\mathbf{k})=$

$$
=\sqrt{3+2 \cos \left(k_{x} a_{0} \sqrt{3}\right)+4 \cos \left(k_{x} a_{0} \sqrt{3} / 2\right) \cos \left(3 k_{y} a_{0} / 2\right)} .
$$

Здесь $\mathbf{k}=\left(k_{x}, k_{y}\right)$ - волновой вектор для движения электрона в плоскости листа графена, $a_{0}$ - расстояния между ближайшими соседями в графене; $\omega$ - энергетическая переменная, $t$ - энергия перехода электрона между ближайшими соседями в графене (кинетический обмен),

$$
\begin{gathered}
\Omega_{A(B)}^{\sigma}=\omega-\varepsilon_{\sigma}(\omega) \mp \Delta_{\sigma}(\omega), \quad 2 \varepsilon_{\sigma}(\omega)=\left(\tilde{\varepsilon}_{A \sigma}+\tilde{\varepsilon}_{B \sigma}\right), \\
2 \Delta_{\sigma}(\omega)=\left(\tilde{\varepsilon}_{A \sigma}+\tilde{\varepsilon}_{B \sigma}\right), \quad \tilde{\varepsilon}_{A(B)_{\sigma}}=\varepsilon_{A(B)_{\sigma}}+\Lambda(\omega)
\end{gathered}
$$

где $\sigma$ - спиновый индекс; функции уширения и сдвига равны соответственно

$$
\begin{gathered}
\Gamma(\omega)=\pi V^{2} \rho_{\mathrm{sub}}(\omega) \\
\Lambda(\omega)=V^{2} P \int_{-\infty}^{\infty} d \omega^{\prime} \rho_{\mathrm{sub}}\left(\omega^{\prime}\right) /\left(\omega-\omega^{\prime}\right),
\end{gathered}
$$

где $\rho_{\text {sub }}(\omega)$ - плотность состояния подложки, $V-$ матричный элемент взаимодействия графен-подложка, $P$ - символ главного значения.

Электронный спектр системы определяется из уравнения $\Omega_{A}^{\sigma} \Omega_{B}^{\sigma}=t^{2} f^{2}(\mathbf{k})$ (действительные полюса функции Грина (1)), что дает

$$
\begin{aligned}
& E_{ \pm}^{\sigma}(\omega, \mathbf{k})=\varepsilon_{a}(\omega) \pm R_{\sigma}(\omega, \mathbf{k}) \\
& R_{\sigma}(\omega, \mathbf{k})=\sqrt{\Delta_{\sigma}^{2}(\omega)+t^{2} f^{2}(\mathbf{k})}
\end{aligned}
$$

Знак минус в (3) соответствует валентной $\pi$-зоне, знак плюс $-\pi^{*}$-зоне проводимости.

В соответствии с результатами $[9,10]$, где для функции $f(\mathbf{k})$ использовалось низкоэнергетическое приближение $f(\mathbf{k}) \approx 3 a_{0}|\mathbf{k}| / 2 \quad[1]$, запишем общее выражение для плотности состояний плоского листа ЭГ на полупроводнике в виде

$$
\begin{aligned}
\rho_{\sigma}^{\mathrm{EG}}(\omega)= & \frac{\Gamma(\omega)}{\pi \xi^{2}} \ln \frac{\left|\xi^{4}+B_{\sigma} \xi^{2}+C_{\sigma}\right|}{C_{\sigma}} \\
& +\frac{2 \tilde{\Omega}_{\sigma}}{\pi \xi^{2}}\left(\arctan \frac{2 \xi^{2}+B_{\sigma}}{4 \Gamma(\omega) \tilde{\Omega}_{\sigma}}-\arctan \frac{B_{\sigma}}{4 \Gamma(\omega) \tilde{\Omega}_{\sigma}}\right) .
\end{aligned}
$$

Здесь $\tilde{\Omega}_{\sigma}=\omega-\tilde{\varepsilon}_{\sigma}, 2 \tilde{\varepsilon}_{\sigma}=\left(\tilde{\varepsilon}_{A \sigma}+\tilde{\varepsilon}_{B \sigma}\right), 2 \Delta_{\sigma}=\left(\tilde{\varepsilon}_{A \sigma}-\tilde{\varepsilon}_{B \sigma}\right)$, $\xi=t \sqrt{2 \pi \sqrt{3}}, \quad G_{\sigma}=-2\left(\tilde{\Omega}_{\sigma}^{2}-\Delta_{\sigma}^{2}-\Gamma_{s c}^{2}(\omega)\right), \quad C_{\sigma}=$ $=\left(\tilde{\Omega}_{\sigma}^{2}-\Delta_{\sigma}^{2}\right)^{2}+\Gamma_{s c}^{2}(\omega)\left(\Gamma_{s c}^{2}(\omega)+2 \Delta_{\sigma}^{2}+2 \tilde{\Omega}_{\sigma}^{2}\right)$.

Энергетические уровни $\varepsilon_{A \sigma}$ и $\varepsilon B \sigma$ атомов подрешеток определяются как

$$
\begin{aligned}
& \tilde{\varepsilon}_{A \uparrow, \downarrow}=\varepsilon_{0}+\Lambda(\omega)+U(a \mp b+c \mp d) / 2+z G(a-c), \\
& \tilde{\varepsilon}_{B \uparrow, \downarrow}=\varepsilon_{0}+\Lambda(\omega)+U(a \mp b-c \pm d) / 2+z G(a+c),
\end{aligned}
$$

где $z=3$ - число ближайших соседей в графене; параметры $a, b, c$ и $d$ связаны с числами заполнения атомов подрешеток $n_{A \sigma}$ и $n_{B \sigma}$ выражениями

$2 a=n_{A \uparrow}+n_{A \downarrow}+n_{B \uparrow}+n_{B \downarrow}, 2 b=n_{A \uparrow}-n_{A \downarrow}+n_{B \uparrow}-n_{B \downarrow}$,
$2 c=n_{A \uparrow}+n_{A \downarrow}-n_{B \uparrow}-n_{B \downarrow}, 2 d=n_{A \uparrow}-n_{A \downarrow}-n_{B \uparrow}+n_{B \downarrow}$,

так что

$$
n_{A \uparrow, \downarrow}=(a \pm b+c \pm d) / 2, n_{B \uparrow, \downarrow}=(a \pm b-c \mp d) / 2 .
$$

Энергия системы есть

$$
\begin{aligned}
E= & \sum_{\alpha, \sigma} \varepsilon_{\alpha \sigma} \hat{n}_{\alpha \sigma}-U \sum_{\alpha} n_{\alpha \uparrow} n_{\alpha \downarrow}-z G n_{A} n_{B} \\
& +\sum_{\alpha, \sigma} \int_{-\infty}^{\varepsilon_{\mathrm{F}}-\varepsilon_{\alpha \sigma}} \Omega_{\sigma} \rho_{\alpha}^{\sigma}\left(\Omega_{\sigma}\right) d \Omega_{\sigma}+E_{\mathrm{sub}},
\end{aligned}
$$

где $\varepsilon_{\mathrm{F}}-$ уровень Ферми, $\rho_{\alpha}^{\sigma}\left(\Omega_{\sigma}\right)-$ плотность состояний для $\alpha=(A, B)$-подрешетки (на атом), $E_{\mathrm{sub}}-$ вклад субстрата в энергию системы.

Для конкретизации функций уширения $\Gamma(\omega)$ и сдвига $\Lambda(\omega)$ необходимо задать плотность состояний полупроводниковой подложки $\rho_{\text {sub }}(\omega)$. Выберем для этого модель Халдейна-Андерсона (см., например, [22,23]), в соответствии с которой

$$
\rho_{\text {sub }}(\omega)= \begin{cases}\rho_{s c}, & |\omega|>E_{g} / 2, \\ 0, & |\omega| \leq E_{g} / 2,\end{cases}
$$

где $\rho_{s c}=$ const, нуль энергии помещен в центр запрещенной зоны шириной $E_{g}$. Тогда функции уширения $\Gamma(\omega)=\Gamma_{s c}=\pi V^{2} \rho_{s c}$ при $|\omega|>E_{g} / 2$ и $\Gamma(\omega)=0$ при $|\omega| \leq E_{g} / 2$. Функция сдвига $\Lambda(\omega)$ есть

$$
\Lambda(\omega)=\frac{\Gamma_{s c}}{\pi} \ln \left|\frac{\omega-E_{g} / 2}{\omega+E_{g} / 2}\right| .
$$

Набор формул (1)-(9) полностью определяет поставленную задачу. Плотности состояний вида (4) для раз- 
личных соотношений параметров задачи рассмотрены в [9,10,22,23] (см. также ссылки, приведенные в этих работах). Аналогично [9] рассмотрим для иллюстрации ряд частных случаев.

\section{2. Гетеропереходы графен-полупроводник}

Рассмотрим для начала СГ, положив $V=0$. При этом функции $\Gamma(\omega)$ и $\Lambda(\omega)$ тождественно обращаются в нуль, получаем плотность состояний СГ:

$$
\rho_{\sigma}^{\mathrm{FG}}\left(\Omega_{\sigma}\right)= \begin{cases}\frac{2\left|\Omega_{\sigma}\right|}{\xi^{2}}, & \bar{R}_{\sigma} \geq\left|\Omega_{\sigma}\right| \geq\left|\Delta_{\sigma}\right|, \\ 0, & \left|\Omega_{\sigma}\right|<\left|\Delta_{\sigma}\right|,\left|\Omega_{\sigma}\right|>\bar{R}_{\sigma},\end{cases}
$$

где $\Omega_{\sigma}=\omega-\varepsilon_{\sigma}, 2 \varepsilon_{\sigma}=\left(\varepsilon_{A \sigma}+\varepsilon_{B \sigma}\right), 2 \Delta_{\sigma}=\left(\varepsilon_{A \sigma}-\varepsilon_{B \sigma}\right)$, $\bar{R}_{\sigma}=\sqrt{\xi^{2}+\Delta_{\sigma}^{2}}$ и $\varepsilon_{A(B) \sigma}=\tilde{\varepsilon}_{A(B) \sigma}$ при $\Lambda(\omega)=0$ (см. [9]). Полагая, как и в [9], $\varepsilon^{*} \equiv \varepsilon_{0}+a(U / 2+z G)=\varepsilon_{\mathrm{F}}$, получим $a=1$ и $n_{A, B}=1 \pm c$. Когда суммарная намагниченность элементарной ячейки ЭГ равна нулю (т.е. $b=0$ ), получим для спиновых моментов $m_{A, B}=n_{A \uparrow, B \uparrow}-n_{A \downarrow, B \downarrow}$ равенство $m_{A}=-m_{B}=d$, соответствующее антиферромагнитному состоянию. При этом фазовая диаграмма СГ содержит три области [9]: однородное по заряду и нулевому спину бесщелевое ПМ состояние, характеризуемое законом дисперсии $E_{ \pm}^{\sigma}(\mathbf{k})=$ $= \pm t|f(\mathbf{k})|$; ВСП состояние, отвечающее закону дисперсии $E_{ \pm}^{\sigma}(\mathbf{k})= \pm \sqrt{(d U / 2)^{2}+t^{2} f^{2}(\mathbf{k})} ;$ В3П состояние с законом дисперсии $E_{ \pm}^{\sigma}(\mathbf{k})= \pm \sqrt{c^{2}(U / 2-z G)^{2}+t^{2} f^{2}(\mathbf{k})}$. Легко видеть, что граница между ВСП и ВЗП состояниями определяется условием $z G=U$.

Перейдем теперь к ЭГ. На рис. 1 работы [10] схематически представлены различные случаи взаимного расположения энергетических зон на контакте графеноподобного соединения и полупроводниковой подложки в отсутствие взаимодействия между ними. Эти энергетические диаграммы являются аналогами обычных полупроводниковых гетеропереходов [24].

Начнем со случая разорванного (broken) гетероперехода (рис. $1, d$ работы $[10]$, гетеропереход типа III). Ясно, что в пределе $\left|\varepsilon_{\sigma}\right| \gg E_{g}$ имеем по сути подробно рассмотренный в [9] случай ЭГ на металле. Действительно, согласно (8), плотность состояний полупроводниковой подложки в этой энергетической области равна константе, как и для случая металлического субстрата в [9]. Легко показать, что в данном пределе имеет место большой переход заряда. Действительно, при $\varepsilon_{\sigma}<-E_{g}$ электрон переходит из валентной зоны подложки в зону проводимости ЭГ. При $\varepsilon_{\sigma}>E_{g}$ осуществляется обратный процесс: электрон из валентной зоны ЭГ туннелирует в зону проводимости подложки. Таким образом, как и в [9], допирование ЭГ приводит к уширению области ПМ состояния, причем эффект не зависит от знака свободных носителей, вносимых в ЭГ подложкой.

Рассмотрим этот случай подробнее. Так как $|\Lambda(\omega)| \propto|\omega|^{-1}$, положим $\Lambda(\omega)=0$ и перепишем систе- му уравнений (27) работы [9] в виде

$$
\begin{aligned}
& -\Gamma_{s c} \tan [\pi(v+c+d) / 2]=\delta+U(c-d) / 2-c z G, \\
& -\Gamma_{s c} \tan [\pi(v+c-d) / 2]=\delta+U(c+d) / 2-c z G,
\end{aligned}
$$

где $\delta=\varepsilon^{*}-\varepsilon_{\mathrm{F}}$. Будем считать $|\delta| \gg E_{g}, a=1+v$, $|v| \sim 1$, причем при $\delta>0$ имеем $v \sim-1$, при $\delta<0-$ $v \sim 1$. Если в обоих уравнениях (11) правые части положительны и велики, то $v+c+d \sim-1$ и $v+c-d \sim-1$. Аналогично, если правые части уравнений (11) отрицательны и велики по модулю, то $v+c+d \sim 1$ и $v+c-d \sim 1$. Отсюда следует, что $c=d=0$, т. е. для ЭГ реализуется аналог ПМ состояния СГ. Однако это уже не дираковский ПМ, характеризуемый нулевой плотностью состояний в точке Дирака и линейной зависимостью от энергии вблизи нее. Здесь плотность состояний в точке Дирака конечна, а зависимость от энергии в ее окрестности нелинейна. Таким образом, в нулевом приближении в пределе $\left|\varepsilon_{\sigma}\right| \gg E_{g}$ отличие металлической подложки от полупроводниковой сводится к замене $\Gamma_{m}$ на $\Gamma_{s c}$.

Перейдем теперь к охватывающим (straddling) гетеропереходам типа I (рис. $1, a, b$ работы [9]). В случае ЭГ в области энергий запрещенной зоны $|\omega| \leq E_{g} / 2$ функция $\Gamma(\omega)$ тождественно обращается в нуль, тогда как функция сдвига $\Lambda(\omega)$ остается конечной и равной нулю лишь при $\omega=0$ (см. (9)). Таким образом, первое слагаемое в выражении (4) исчезает. Исчезает и второе слагаемое в (4), если числители аргументов арктангенсов имеют один и тот же знак, т.е. удовлетворяют неравенству $B_{\sigma}\left(2 \xi^{2}+B_{\sigma}\right)>0$. При этом плотность состояний ЭГ тождественно равна нулю. Приведенное неравенство удовлетворяется в случае

$$
\left|\tilde{\Omega}_{\sigma}\right|<\left|\Delta_{\sigma}\right|, \quad\left|\tilde{\Omega}_{\sigma}\right|>\bar{R}_{\sigma},
$$

где

$$
\tilde{\Omega}_{\sigma}=\omega-\varepsilon_{\sigma}-\Lambda_{s c}(\omega) .
$$

В области энергий, удовлетворяющих условию $\left|\Delta_{\sigma}\right| \leq\left|\tilde{\Omega}_{\sigma}\right| \leq \bar{R}_{\sigma}$, плотность состояний (4) совпадает с выражением (10) при замене в нем $\Omega_{\sigma}=\omega-\varepsilon_{\sigma}$ на $\tilde{\Omega}_{\sigma}=\Omega_{\sigma}-\Lambda(\omega)$. В пределе $|\omega| \ll E_{g}$ функция сдвига $\Lambda(\omega) \approx-\omega \Gamma_{s c} / \pi E_{g}$. Пренебрегая в нулевом приближении сдвигом $\Lambda(\omega)$, приходим к случаю СГ.

Ступенчатый (staggered) гетеропереход типа II (рис. 1, с работы [9]) занимает промежуточную позицию между гетеропереходами I и III типов и здесь специально не рассматривается. Таким образом, несколько упрощая, можно утверждать, что в случае полупроводниковой подложки в зависимости от параметров задачи плотность состояний ЭГ может изменяться от плотности состояний СГ до плотности состояний ЭГ на металле. Более подробное рассмотрение гетеропереходов в рамках кластерной модели $[9,10]$ дано в Приложении. Там же приведены некоторые численные оценки параметров. 


\section{3. Электрон-фононное взаимодействие}

Анализ роли электрон-фононного взаимодействия, проведенный в работе [21] в рамках расширенной модели Холстейна-Хаббарда (ЕННМ), показал, что теперь вместо выражения (5) для энергетических уровней $\varepsilon_{A \sigma}$ и $\varepsilon_{B \sigma}$ получим

$$
\begin{gathered}
\tilde{\varepsilon}_{A \uparrow, \downarrow}=\varepsilon_{0}+\Lambda(\omega)+U(a \mp b+c \mp d) / 2 \\
+z G(a-c)-\lambda(a+c), \\
\tilde{\varepsilon}_{B \uparrow, \downarrow}=\varepsilon_{0}+\Lambda(\omega)+U(a \mp b-c \pm d) / 2 \\
+z G(a+c)-\lambda(a-c) .
\end{gathered}
$$

Здесь суммарная константа электрон-фононного взаимодействия $\lambda=\lambda_{\|}+\lambda_{\perp}$, где $\lambda_{\|}$и $\lambda_{\perp}-$ константы электрон-фононного взаимодействия для колебаний атомов углерода в плоскости графенового листа и колебаний графенового листа относительно подложки.

Как показано в [21], константа электрон-фононной связи СГ для длинноволнового оптического фонона с энергией $\omega_{\|}$равна $\lambda_{\|}=w_{\|}^{2} / M \omega_{\|}^{2}$, где $M-$ масса атома углерода и деформационная константа $w_{\|}=2 t / a_{0}$. При-

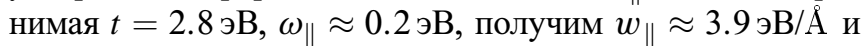
$\lambda_{\|} \approx 0.13$ эВ [21].

Перейдем теперь к оценке деформационной константы $w_{\perp}$ и константы электрон-фононной связи $\lambda_{\perp}=w_{\perp}^{2} / M \omega_{\perp}^{2}$ для колебаний с частотой $\omega_{\perp}$ листа графена (как целого) относительно полупроводниковой подложки. В соответствии с методом связывающих орбиталей Харрисона [25-27], матричный элемент ковалентной связи атома углерода графена с атомом подложки $V=\eta_{b}\left(\hbar^{2} / m_{0} a_{\perp}^{2}\right)$, где $a_{\perp}-$ pacстояние графен-подложка, $m_{0}-$ масса свободного электрона, $\hbar$ - постоянная Планка, $\eta_{b}-$ численный коэффициент для $\sigma$-связи, зависящий от образующих эту связь гибридизованных орбиталей. Для $s p^{3}$-орбиталей $\eta_{b}=3.22[26,27]$. Для $\sigma$-связи $p_{z}$-орбитали атома графена с $s p^{3}$-орбиталью подложки получим $\eta_{b}=2.63$ [28]. Полагая, как и в [21], $w_{\perp}=-\partial V / \partial a_{\perp}, \quad$ получаем $w_{\perp}=2 V / a_{\perp}$. Принимая, согласно расчетам [29], $a_{\perp}=2 \AA$, получим в первом случае $V \approx 6$ эВ и $w_{\perp} \approx 3$ эВ/A, а во втором $-V \approx 5$ эВ и $w_{\perp} \approx 2.5$ эВ $/ \AA$. По данным эксперимента [30], однако, $a_{\perp} \approx 3 \AA$. Такому расстоянию отвечает уже не ковалентное, а ван-дерваальсово взаимодействие. Используя результаты работы [21], получим $w_{\perp}=12 D / a_{\perp}$, где $D-$ величина энергии ван-дер-ваальсовой связи графена с подложкой. В случае ковалентного взаимодействия уместно говорить о буферном слое, в случае взаимодействия Вандер-Ваальса - о квазисвободном графене [21].

Оценим теперь центральную силовую константу $k_{0 \perp}=M \omega_{\perp}^{2}[21,31]$. Воспользовавшись результатами работы [32] и пренебрегая для простоты оценки ионностью и металличностью, для ковалентной связи запишем $k_{0 \perp} \approx 4 V / a_{\perp}^{2}$, откуда $k_{0 \perp} \approx 6$ эВ/ $\AA^{2}$ для $\sigma$-связи, образованной $s p^{3}$-орбиталями атома графена и подложки, и

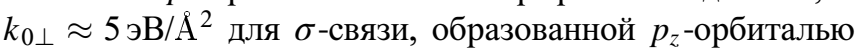
атома графена и $s p^{3}$-орбиталью подложки. Для ван-дерваальсова взаимодействия $k_{0 \perp}=72 D / a_{\perp}^{2}$ [21]. Отсюда $\lambda_{\perp} \approx w_{\perp}^{2} / k_{0 \perp}$, так что 1.50 и 1.25 эВ в случае ковалентной связи $s p^{3}$ - и $p_{z}$-орбиталей графена с $s p^{3}$-орбиталью подложки и $\lambda_{\perp}=2 D$ в случае взаимодействия Вандер-Ваальса. По порядку величины энергия $D$ равна нескольким десятым долям эВ.

\section{4. Обсуждение результатов и заключение}

Итак, применение ЕНМ к полупроводниковой подложке показало, что в общем случае фазовая диаграмма ЭГ качественно та же, что и в случае металлической подложки [9]. Параметры модели для этих субстратов, естественно, отличаются, что ведет к сдвигу границ между областями фазовой диаграммы. То же можно сказать и об использовании ЕННМ для полупроводникового субстрата. Так, например, легко показать, что в случае $a=1, b=0$ роль электрон-фононного взаимодействия сводится к перенормировке межцентрового отталкивания $G$, переходящего в $G^{*}=G+\lambda / z$. Так как $U \approx 9.3$ эВ и $G \approx 5.5$ эВ [33], для наиболее интересного случая квазисвободного графена получаем $U, G \gg \lambda / z$, т.е. тот же результат, что и для графена на металле [21]. Более того, для сильной связи графен-подложка $\lambda_{\perp} \approx 1$ эВ (металл) и $1.25-1.50$ эВ (полупроводник). Таким образом, с точки зрения параметров задачи различия малы и все выводы $[9,21]$ относительно диаграмм состояния ЭГ справедливы и в рассматриваемом здесь случае. Отметим, что и в эксперименте [34], где изучался ЭГ на подложках $\mathrm{Ir}$ и $\mathrm{SiC}$, также не упомянуты какиелибо кардинальные различия.

С точки зрения развитой здесь теории, однако, такие различия могут иметь место в случае, когда точка Дирака ЭГ попадает в запрещенную зону полупроводниковой подложки. Если при этом реализуется ВСП или ВЗП состояния (возможные в случае квазисвободного ЭГ [9]), то в спектре и плотности состояний ЭГ появляются щели, как это имеет место в гексагональных графеноподобных слоях (см. рис. 2 и 3 работы [10]). В случае металлической подложки подобные щели невозможны. Отметим в заключение, что модели ЕНМ и ЕННМ применимы и к ЭГ на диэлектрических подложках, таких, например, как $\mathrm{SiO}_{2}$.

\section{Приложсение}

Рассмотрим для упрощения задачи модель шестиатомного кластера, хорошо зарекомендовавшую себя в работе [9] (см. рис. 3). Обобщая результаты [9] на случай полупроводниковой подложки, запишем диагональные 
функции Грина для атомов подрешеток $A$ и $B$ в виде

$$
\begin{aligned}
& \tilde{D}_{A A}^{\sigma}(\omega)=\frac{1}{2}\left(1-C_{\sigma}\right) \tilde{g}_{+}^{\sigma}(\omega)+\frac{1}{2}\left(1+C_{\sigma}\right) \tilde{g}_{-}^{\sigma}(\omega), \\
& \tilde{D}_{B B}^{\sigma}(\omega)=\frac{1}{2}\left(1+C_{\sigma}\right) \tilde{g}_{+}^{\sigma}(\omega)+\frac{1}{2}\left(1-C_{\sigma}\right) \tilde{g}_{-}^{\sigma}(\omega),
\end{aligned}
$$

где

$$
\tilde{g}_{ \pm}^{\sigma}(\omega)=\left(\omega-\tilde{\varepsilon}_{ \pm \sigma}+i \Gamma(\omega)\right)
$$

И

$$
\tilde{\varepsilon}_{ \pm \sigma}=\varepsilon_{\sigma}+\Lambda(\omega) \pm t_{\sigma}, t_{\sigma}=\sqrt{\Delta_{\sigma}^{2}+z t^{2}}, \quad C_{\sigma}=\left|\Delta_{\sigma}\right| / t_{\sigma} .
$$

Соответствующие плотности состояний есть:

$$
\begin{aligned}
& \tilde{\rho}_{A A}^{\sigma}(\omega)=\frac{1}{2}\left(1-C_{\sigma}\right) \tilde{\rho}_{+}^{\sigma}(\omega)+\frac{1}{2}\left(1+C_{\sigma}\right) \tilde{\rho}_{-}^{\sigma}(\omega), \\
& \tilde{\rho}_{B B}^{\sigma}(\omega)=\frac{1}{2}\left(1+C_{\sigma}\right) \tilde{\rho}_{+}^{\sigma}(\omega)+\frac{1}{2}\left(1-C_{\sigma}\right) \tilde{\rho}_{-}^{\sigma}(\omega),
\end{aligned}
$$

где в области разрешенных состояний $|\omega|>E_{g}$ плотности состояний $\tilde{\rho}_{ \pm}^{\sigma}(\omega)$ равны

$$
\tilde{\rho}_{ \pm}^{\sigma}(\omega)=\frac{1}{\pi} \frac{\Gamma_{s c}}{\left(\omega-\tilde{\varepsilon}_{ \pm \sigma}\right)^{2}+\Gamma_{s c}^{2}},
$$

а в области запрещенной зоны $|\omega| \leq E_{g}$ имеем $\tilde{\rho}_{ \pm}^{\sigma}(\omega)=\delta\left(\omega-\tilde{\varepsilon}_{ \pm \sigma}\right)$, где $\delta(.)-.\delta$-функция Дирака.

Рассмотрим для начала ситуацию, когда $\varepsilon^{*}=\varepsilon_{\mathrm{F}}=0$ $(a=1$ и $b=0)$ и $\left|\varepsilon_{\sigma}\right| \gg E_{g}$. Для нахождения чисел заполнения прибегнем к дополнительному упрощению, часто применяемому в теории адсорбции [23], и заменим $\Lambda(\omega)$ и $\Gamma(\omega)$ на $\Lambda\left(\varepsilon_{ \pm \sigma}\right)$ и $\Gamma\left(\varepsilon_{ \pm \sigma}\right)$. Тогда $\Gamma\left(\varepsilon_{ \pm \sigma}\right)=\Gamma_{s c}$. Учитывая, что в области энергий $|\omega| \gg E_{g}$ функция сдвига $\Lambda(\omega) \approx-\Gamma_{s c} E_{g} / \pi \omega$, получим $\tilde{\varepsilon}_{ \pm \sigma} \approx \pm t_{\sigma}^{*}$, где $t_{\sigma}^{*}=t_{\sigma}\left(1-\Gamma_{s c} E_{g} / \pi z t_{\sigma}^{2}\right)$. Полагая, как и в [22], для ковалентной связи графен-подложка $V_{\text {eff }}=V / 3 \sim 2$ эВ и $\rho_{\text {sub }}=0.25$ эВ $^{-1}$, получим $\Gamma_{s c} \approx 3$ эВ. Учтем, что $t \sim 3$ эВ [1] и $E_{g} \sim 3$ эВ (политипы $4 \mathrm{H}, 6 \mathrm{H}$, $8 \mathrm{H}, 15 \mathrm{R}, 18 \mathrm{R}, 21 \mathrm{R}$ карбида кремния [35]). Тогда $\Gamma_{s c} E_{g} / \pi z t^{2} \sim(z \pi)^{-1} \sim 0.1$. В случае ван-дер-ваальсовой связи $\Gamma_{s c} \sim D$, так что $\Gamma_{s c} E_{g} / \pi z t^{2} \ll 1$. Таким образом, все свелось к перенормировке параметра $t_{\sigma}$. Так как $t_{\sigma} \geq z t$, поправку всегда можно считать малой.

Для рассматриваемого случая система уравнений (26) работы [9] переходит в

$$
\begin{aligned}
& \pi(c-d)=2 C_{\downarrow}^{*} \arctan \left(t_{\downarrow}^{*} / \Gamma_{s c}\right), \\
& \pi(c+d)=2 C_{\uparrow}^{*} \arctan \left(t_{\uparrow}^{*} / \Gamma_{s c}\right),
\end{aligned}
$$

где $C_{\sigma}^{*}=\left|\Delta_{\sigma}\right| / t_{\sigma}^{*}, \Delta_{\uparrow, \downarrow}=U(c \mp d) / 2-c z G$. Обобщая результаты [9] на рассматриваемый случай, можем записать, что решение $c \neq 0, d=0$ (неоднородное зарядовое распределение - НЗП) имеет место при выполнении неравенства $2 z G-U>\pi \Gamma_{s c} \eta^{*}$, где $\eta^{*}=\tau \arctan (\tau)$, где $\tau=\left(t^{*} \sqrt{z} / \Gamma_{s c}\right)$ и $t^{*}=t\left(1-\Gamma_{s c} E_{g} / \pi z t^{2}\right)$. Решение $c=0$, $d \neq 0$ (неоднородное спиновое распределение - НСП) возникает при условии $U>\pi \Gamma_{s c} \eta^{*}$. Граница между НЗП и НСП состояниями, соответствующая условию $c=d \neq 0$, отвечает неравенству $U=z G>\pi \Gamma_{s c} \eta_{\max }$, где $\eta_{\max }=\left(t_{\max }^{*} / \Gamma_{s c}\right) / \arctan \left(t_{\max }^{*} / \Gamma_{s c}\right)$ и

$$
t_{\max }^{*}=\sqrt{(z G)^{2}+z\left(t^{*}\right)^{2}}\left\{1-\Gamma_{s c} E_{g} / \pi\left[(z G)^{2}+z\left(t^{*}\right)^{2}\right]\right\} .
$$

Нетрудно видеть, что положив $E_{g}=0$, получим результаты работы [9] для металлической подложки. Соответствующая энергетическая диаграмма изображена на рис. 4 работы [9], в которой, однако, $\Gamma_{m}$ и $\eta$ нужно заменить на $\Gamma_{s c}$ и $\eta^{*}$. Если же положить $t=0$, то приходим к частному случаю, фазовая диаграмма которого представлена на рис. 2 работы [9] при условии замены $\Gamma_{m}$ на $\Gamma_{s c}$. И наконец, при $\Gamma_{s c} \rightarrow 0$ переходим к случаю СГ с учетом межатомного $t$-взаимодействия, но не в зонном варианте, а в кластерной модели. Тогда для существования НСП необходимо выполнение неравенства $U>2 \sqrt{z} t^{*} \approx 3.5 t^{*}$, для существования НЗП неравенства $2 z G-U>2 \sqrt{z} t^{*}$.

\section{Список литературы}

[1] A.H. Castro Neto, F. Guinea, N.M.R. Peres, K.S. Novoselov, A.K. Geim. Rev. Mod. Phys., 81, 109 (2009).

[2] V.N. Kotov, B. Uchoa, V.M. Pereira, A.H. Castro Neto, F. Guinea. Rev. Mod. Phys., 84, 1067 (2012).

[3] N. Swain, P. Majumdar. arXive: 1610.00695.

[4] M.V. Ulybyshev, P.V. Buividovich, M.I. Katsnelson, M.I. Polikarpov. Phys. Rev. Lett., 111, 056801 (2013).

[5] L. Wang, P. Corboz, M. Troyer. New J. Phys., 16, 103008 (2014).

[6] M. Hohenadler, F.P. Toldin, I.F. Herbut, F.F. Assaad. Phys. Rev. B, 90, 085146 (2014).

[7] W. Wu, A.-M.S. Tremblay. Phys. Rev. B, 89, 205128 (2014).

[8] L. Classen, I.F. Herbut, L. Janssen, M.M. Scherer. Phys. Rev. B, 92, 035429 (2015).

[9] С.Ю. Давыдов. ФТТ, 59, 1650 (2017).

[10] С.Ю. Давыдов. ФТТ, 58, 1182 (2016).

[11] M. Hohenadler, W. von der Linden. Phys. Rev. B, 71, 184309 (2005).

[12] M. Berciu. Phys. Rev. B, 75, 081101R (2007).

[13] R.P. Hardikar, R.T. Clay. Phys. Rev. B, 75, 245103 (2007).

[14] S. Kumar, J. van den Brink. Phys. Rev. B, 78, 155123 (2008).

[15] Y. Murakami, P. Werner, N. Tsuji, H. Aoki. Phys. Rev. B, 88, 125126 (2013).

[16] M. Chakraborty, M. Tezuka, B.I. Min. Phys. Rev. B, 89, 035146 (2014).

[17] P. Werner, M. Eckstein. Europhys. Lett., 109, 37002 (2015).

[18] S. Pradhan, G.V. Pai. Phys. Rev. B, 92, 165124 (2015).

[19] I.V. Sankar, A. Chatterjee. Physica B, 489, 17 (2016).

[20] T. Miyao. arXive: $1402.5202 ; 1610.09039$.

[21] С.Ю. Давыдов. ФТП, принято к печати.

[22] С.Ю. Давыдов. ФТП, 47, 97 (2013).

[23] С.Ю. Давыдов. Теория адсорбции: метод модельных гамильтонианов (СПб., Изд-во СПбГЭТУ ЛЭТИ, 2013). Электронный адрес: twirpx.com/file/1596114/ 2007. 96 с. twirpx.com/file/1014608/

[24] Ф. Бехштедт, Р. Эндерлайн. Поверхности и границы раздела полупроводников (М., Мир, 1990). 
[25] У. Харрисон. Электронная структура и свойства твердых тел (М., Мир, 1983) т. 1.

[26] W.A. Harrison. Phys. Rev. B, 31, 2121 (1985).

[27] С.Ю. Давыдов, О.В. Посредник. Метод связывающих орбиталей в теории полупроводников. Учеб. пособие (СПб., Изд-во СПбГЭТУ „ЛЭТИ“, 2007). twirpx.com/file/1014608/

[28] С.Ю. Давыдов, Г.И. Сабирова. Письма ЖТФ, 37 (11), 51 (2011).

[29] A. Mattausch, O. Pankratov. Phys. Rev. Lett., 99, 076802 (2007).

[30] J. Borysiuk, J. Sołtys, R. Bozek, J. Piechota, S. Krukowski, W. Strupinski, J.M. Baranowski, R. Stepniewski. Phys. Rev. B, 85, 045426 (2012).

[31] С.Ю. Давыдов. ФТТ, 59, 3, 610 (2017).

[32] С.Ю. Давыдов, О.В. Посредник. ФТТ, 57, 819 (2015).

[33] T.O. Wehling, E. Sasioglu, C. Friedrich, A.I. Lichtenstein, M.I. Katsnelson, S. Blügel. Phys. Rev. B, 106, 236805 (2011).

[34] J.C. Johannsen, S. Ulstrup, M. Bianchi, R. Hatch, D. Guan, F. Mazzola, L. Hornekær, F. Fromm, C. Raidel, T. Seyller, P. Hofmann. J. Phys.: Condens. Matter, 25, 094001 (2013).

[35] В.И. Гавриленко, А.М. Грехов, Д.В. Корбутяк, В.Г. Литовченко. Оптические свойства полупроводников. Справочник (Киев, Наук. думка, 1987).

Редактор Г.А. Оганесян

\section{Electron-electron and electron-phonon interactions in graphene on semiconducting substrate: Simple estimations}

\section{S.Yu. Davydov}

loffe Institute,

194021 St. Petersburg, Russia

National Research University of Information

Technologies, Mechanics and Optics,

197101 St. Petersburg, Russia

Abstract Within the scopes of the extended Hubbard and Holstein-Hubbard models problem on epitaxial graphene fabricated on semiconducting substrate is considered. HaldaneAnderson model is used for the substrate density of states. Three regions of phase diagram are considered: spin-density wave (SDW), charge-density wave (CDW), and spin and charge uniform paramagnetic distribution. For the number of particular cases the similarities and differences of the graphene on metal and semiconductor electronic states. It is demonstrated that the main distinction arises in the case when the graphene's Dirac point lies within the substrate energy gap. Numerical estimations are fulfilled for the SiC substrate. 